\title{
Variability of structural and functional traits of vascular tissues in tomato: modeling, histology and flow-MRI investigations
}

\author{
Jeanne Simon ${ }^{1}$, Maïda Cardoso ${ }^{2}$, Béatrice Brunel ${ }^{1}$, Eric Alibert ${ }^{2}$, Christelle Baptiste $^{3}$, \\ Marc Lartaud $^{3}$, Jean-Luc Verdeil ${ }^{3}$, Gilles Vercambre ${ }^{1}$, Christophe Goze-Bac ${ }^{2}$, and Nadia \\ $\operatorname{Bertin}^{1,1}$ \\ ${ }^{1}$ INRAE \\ ${ }^{2}$ Université de Montpellier \\ ${ }^{3}$ CIRAD Centre de Montpellier
}

August 28, 2020

\begin{abstract}
Vascular tissues are main routes of resource transport, which are crucial for the growth of fleshy fruit. Very few quantitative data of the total and active areas of xylem and phloem are available for herbaceous plants and their variabilities are unknown. In this study, histological approach and process-based modeling of tomato fruit growth were combined to evaluate the potential contribution of the pedicel anatomy to fruit mass variations. Eleven genotypes were described and the impact of water deficit was studied depending on stress intensity and stage of application. In parallel, MRI experiments allowed to better understand the source of variability in xylem flow along the main stem. Our results suggested that the genetic and water deficit-induced variations in the areas of vascular tissues in the pedicel partly contributed to fruit mass variability. Flow-MRI appeared to be a complementary non-destructive method to phenotype vascular tissues. Whereas the flow velocity in active xylem vessels was rather stable along the main stem, the decrease in the number of active vessels strongly reduces the effective flow from the bottom to the top of the plant. Fruitful interactions between modeling, histology and flow-MRI are promising and worth exploring, to predict water fluxes within plant architecture.
\end{abstract}

\section{KEYWORDS}

Solanum lycopersicum, xylem, phloem, histology, water deficit, genotypic variability, Virtual Fruit model, flow-MRI

\section{ACKNOWLEDGEMENTS}

We thank Guillaume Garcia, Thibault Crouzet and Pierre Valsesia for their valuable contributions to this work.

Variability of structural and functional traits of vascular tissues in tomato: modeling, histology and flow-MRI investigations

Jeanne Simon ${ }^{1,2^{*}}$, Maïda Cardoso ${ }^{3}$, Béatrice Brunel ${ }^{1}$, Eric Alibert ${ }^{2}$, Christelle Baptiste ${ }^{4}$, Marc Lartaud ${ }^{4}$, Jean-Luc Verdeil ${ }^{4}$, Gilles Vercambre ${ }^{1}$, Christophe Goze-Bac ${ }^{2}$, Nadia Bertin ${ }^{1}$

${ }^{1}$ INRAE UR1115 Plantes et Systèmes de culture Horticoles - Site Agroparc - 84914, Avignon, France, ${ }^{2}$ Université Montpellier-CNRS, Laboratoire Charles Coulomb UMR 5221, F-34095, Montpellier, France, ${ }^{3}$ Université Montpellier, BNIF Imaging facility, F-34095, Montpellier, France; ${ }^{4}$ UMR AGAP, CIRAD, Montpellier, France

Corresponding author 


\begin{abstract}
Vascular tissues are main routes of resource transport, which are crucial for the growth of fleshy fruit. Very few quantitative data of the total and active areas of xylem and phloem are available for herbaceous plants and their variabilities are unknown. In this study, histological approach and process-based modeling of tomato fruit growth were combined to evaluate the potential contribution of the pedicel anatomy to fruit mass variations. Eleven genotypes were described and the impact of water deficit was studied depending on stress intensity and stage of application. In parallel, MRI experiments allowed to better understand the source of variability in xylem flow along the main stem. Our results suggested that the genetic and water deficitinduced variations in the areas of vascular tissues in the pedicel partly contributed to fruit mass variability. Flow-MRI appeared to be a complementary non-destructive method to phenotype vascular tissues. Whereas the flow velocity in active xylem vessels was rather stable along the main stem, the decrease in the number of active vessels strongly reduces the effective flow from the bottom to the top of the plant. Fruitful interactions between modeling, histology and flow-MRI are promising and worth exploring, to predict water fluxes within plant architecture.
\end{abstract}

Keywords: Solanum lycopersicum, xylem, phloem, histology, water deficit, genotypic variability, Virtual Fruit model, flow-MRI

\title{
1 - INTRODUCTION
}

Water and nutrient fluxes from source organs (leaves, roots) to sink organs (fruit, root tip, apex...) determine the growth and quality of fleshy fruits, such as tomato. Resources are transported through the xylem vessels and through the phloem sieve elements, which drive respectively, the transport of water and minerals from roots to leaves and fruits, and the translocation of sugar and amino acids from leaves to sink organs (Ho, 1996). Vascular tissues also transport molecules implicated in the signalization on long distances, which play an important role in response to stress (Lough and Lucas 2006 ; Spiegelman et al., 2015).

Many studies based on destructive or indirect methods such as isotopic labeling, balance method, heat girdling, have shown that the phloem tissues transport 80 to $90 \%$ of water imported by tomato fruit, while the remaining 10 to $20 \%$ is transported by the xylem (Ho et al., 1987; Van Bel, 1990; Guichard et al., 2005). However, contrasting results were observed by Magnetic Resonance Imaging (MRI) (Windt et al., 2009). Regarding photoassimilates, tomato fruit trusses are mostly supplied by the leaves below the truss through the internal phloem and to a lesser extent by leaves above the truss, through the external phloem, suggesting upward and downward movement in the respective tissues (Bonnemain, 1968; André et al., 1999). MRI measurements (Windt et al., 2009) also advocated the low contribution of the external phloem to fruit water supply. However, long distance transport of assimilates between remote sources and sinks has been demonstrated by truss pruning experiments (Slack and Calvert 1977; Heuvelink, 1995), thus suggesting that both internal and external phloem strands are functional for resource allocation to fruits.

The vascular system intensively develops during the early phase of flower bud and fruit development on both sides of the abscission zone (AZ) and the hydraulic conductance increases in the peduncle and pedicel parts during fruit development, due to secondary growth and formation of new vessels as reflected by the increase in pedicel and peduncle diameters (André et al., 1999; van Ieperen et al., 2003; Rancic et al., 2010). At the level of the $\mathrm{AZ}$ of the pedicel, the differentiation is delayed as well as the xylem lignification, and the higher hydraulic resistance is attributed to the lower number of differentiated vessels with reduced cross-sectional area, relatively to the rest of the pedicel (Lee, 1989; André et al. 1999; van Ieperen et al., 2003).

The role of the AZ resistance in preventing xylem backflow in period of water deficit (WD) has been widely discussed (Lee, 1989; Guichard et al., 2001; Malone and Andrews, 2001). Indeed, the hydraulic resistance of the pedicel AZ increases in plants grown under WD on the contrary to the resistance on both sides of the AZ, in which new secondary vessels are formed (van Ieperen et al. 2003). In agreement, WD reduces the pedicel xylem area, but increases the proportion of conducting vessels before and after the AZ (Rancic et al., 2008). Malone and Andrews (2001) confirmed the high hydraulic resistance between stem and fruit, but showed that xylem is functional and continuous throughout the tomato pedicel and estimated that more 
than $90 \%$ of the hydraulic resistance would occur within the fruit pericarp.

On their whole, these studies point out the need for an improved characterization of the structural and functional properties of vascular tissues and their variabilities, in order to understand and predict water fluxes between stem and fruit. In this perspective, process-based models could help integrating knowledge to analyze the effects of environmental and genetic factors on fruit growth. A modeling approach of water flow through the pedicel and within the fruit suggested that water importation in the young growing tomato could be limited mainly by the pedicel resistance and the phloem conductivity, linked to sap viscosity (Bussières 2002 ; Bussières et al., 2011). In agreement, a study on the design of tomato fruit ideotypes adapted to WD based on the Virtual Fruit model (Fishman and Génard, 1998 ; Liu et al., 2007) led to the hypothesis that the conductance of the vascular tissues in the pedicel could be an interesting adaptation trait (Constantinescu et al., 2016). The Virtual Fruit model is a biophysical model of fruit expansion, which has been further extended to describe the longitudinal water fluxes in the pedicel and both longitudinal and radial water fluxes in the fruit (Hall et al., 2013). Using this model, Constantinescu et al. (2020) suggested that phloem-to-xylem bi-directional exchanges of water may regulate the daily influx of sugar to the fruit and buffer the variations of sugars in the pedicel, while xylem-to-apoplast recirculation of water through the fruit phloem may reduce xylem backflow at midday. Yet in the Virtual Fruit model, the xylem and phloem conductances are parameterized in a rather empirical way, assuming proportionality between fruit surface and xylem and phloem conductances, and constant xylem and phloem conductivities in the pedicel. Experimental data is missing to evaluate and validate such hypotheses. More generally, vascular tissue area, conductivity and viscosity interact, but none of these variables are easily measured, and they are usually predicted empirically in growth models, while they become overriding to simulated fluxes in stress conditions. Combining anatomical observations and flow measurements may help to address the issue of resource transport in the plant. To this aim, MRI has been successfully applied to study water fluxes in plant (Van As, 2007; Windt et al., 2009; Buy et al., 2018).

In the present study, we combined histological observations, process-based modeling and flow-MRI measurements in order to assess variations in the structural and functional properties of vascular tissues in tomato plant during the reproductive period. An image analysis pipeline was developed to measure xylem and phloem cross-sectional areas of the vascular tissues in the pedicel of contrasting genotypes and assess the effect of WD. Then, this variability was implemented in the Virtual Fruit model, in order to evaluate its contribution to variations in fruit fresh and dry masses. The vascular areas were compared in the truss peduncle, fruit pedicel and main plant stem. Comparison of histological and MRI images allowed to estimate the proportion, the diameter and the theoretical conductance of active vessels along the stem.

\section{2 - MATERIAL AND METHODS}

\section{1 - Plant material and growth conditions}

The study investigated 11 contrasting genotypes of tomato (Solanum lycopersicum L.): West Virginia 106 (WVA106) a cherry tomato cultivar, the 8 parent genotypes of the MAGIC TOM population (Cervil, Criollo, Ferum, LA0147, LA1420, Levovil, PlovdivXXIVa (here called Plovdiv), Stupicke Polni Rane (here called Stupicke)) (Pascual et al., 2015 ; Ripoll et al., 2016), the wild-type of the Heinz genotype and the « Jointless » mutant of Heinz with no abscission zone (Mao et al., 2000). All plants were grown in a heated glasshouse $\left(18+/-2{ }^{\circ} \mathrm{C}\right.$ night $/ 22+/-4{ }^{\circ} \mathrm{C}$ day) in Avignon, in $5 \mathrm{~L}$ pots filled with humus (Klasmann, Substrat SP $15 \%$ ) and automatically watered with a commercial nutrient solution (Liquoplant Rose, Plantin, Courthézon, France) in order to match the evaporative demand (control condition).

For the WD experiment, WVA106 plants were grown under three scenarios of irrigation: control, $-50 \%$ of control (light WD) and $-65 \%$ of control (severe WD). In case of severe WD, the reduction was applied either 15 days before the truss anthesis (early severe WD) or 4 days after the truss anthesis (late severe WD). In case of light WD, the reduction was applied 15 days before truss anthesis (early light WD). Treatments were applied on rows of 12 plants surrounded by border plants and each treatment was applied on two different rows (24 plants in total for each treatment). WD intensity was monitored according to previous experiments 
performed under the same glasshouse and genotype (Koch et al., 2018). Soil humidity (measured on soil aliquots) was kept constant until plant harvest at about 1.6 (control), 1.0 (light WD) and 0.6 (severe WD) $\mathrm{gH}_{2} \mathrm{O} \mathrm{g}^{-1}$ of dried soil as described in Koch et al. (2018). The amount and frequency of irrigation increased as plants developed and varied daily depending on potential evapotranspiration (at least four daily irrigations). Side-shoots were removed and trusses were pollinated with a vibrator three times a week. Fruit trusses were pruned to 10 set-fruits per truss so that fruit load was comparable in control and WD. The glasshouse was whitened to avoid thermal stress. The average relative air humidity ranged from $40-50 \%$ at the beginning of the experiment to about $70 \%$.

\section{2 - Pedicel and fruit growth}

The pedicel and the fruit diameters of the second proximal (close to the stem) fruits of trusses that developed concomitantly on all genotypes were measured with a caliper (Digimatic AOS 500-161-30) throughout development (from fruit set to maturity). The pedicel diameter was measured at three positions: at the abscission zone (AZ) level, 5 to $10 \mathrm{~mm}$ before $\mathrm{AZ}$ on the stem side (AZs), and 5 to $10 \mathrm{~mm}$ after $\mathrm{AZ}$ on the fruit side (AZf) (Fig. 1A). At the red ripe stage of the fruit, pedicels were sampled and immediately fixed and processed as described below for histological analysis. The fruits were weighted and dried in a ventilated oven to determine their dry mass and water content.

\section{3 - Histological pipeline to quantify the vascular tissue areas}

A histological pipeline was developed to characterize the vascular elements in truss peduncle, fruit pedicel and main stem (Fig. 1). First, fresh samples were fixed in Clarke's fixative solution (9 volumes of absolute ethanol in 1 volume of acetic acid) to keep the tissue structure (Sharma, 1956). Then, samples were embedded in a 5\% agarose gel (Ref. LE-8200-B Sigma) and transversally cut using a vibratome (Microm HM 650 V). Slices were treated with a bleach solution (10 to $13 \%$ available chlorine) to remove the cell content and increase the contrast among tissues. Histological sections were stained overnight with a FASGA solution diluted at $1 / 7$ (Tolivia and Tolivia, 1987), then rinsed in a $50 \%$ glycerol solution. FASGA mother solution was composed of $2 \mathrm{~mL}$ of Safranine solution at $1 \%, 14 \mathrm{~mL}$ of a Alcian Blue solution at $0.5 \%, 1 \mathrm{~mL}$ of acetic acid, $30 \mathrm{~mL}$ of glycerin and $19.5 \mathrm{~mL}$ of distilled water.

Glass slides were converted into high-resolution digital data with a Nanozoomer RS slide scanner (NanoZoomer Digital Pathology System, Hamamatsu, Japan). A plugin (PHIV_Tomate_toolset) was developed under Fiji (Schindelin et al., 2012) to calculate the number and the area of xylem and phloem vessels. Pedicel, truss peduncle and main stem transversal sections were divided in five anatomical zones corresponding to (from the outer surface to the center): cortex, external phloem, secondary xylem, internal phloem and pith (Fig. 1C, D, E). The masks of the tissues were achieved semi-automatically with the plugin and then corrected manually (if necessary) before calculating the areas of each tissue.

\section{4 - MRI measurements of in vivo fluxes and area of functional xylem vessels}

MRI experiments were performed on a Agilent MRI scanner working at $9.4 \mathrm{~T}$ corresponding to $400 \mathrm{MHz}$ for the ${ }^{1} \mathrm{H}$ nuclei, the most commonly observed atom in MRI experiments, which directly informs on tissue water content and dynamics (Van As, 2007). The MRI experimental set-up on living systems is based on a horizontal superconducting magnet (Magnex Scientific) with a $10 \mathrm{~cm}$ diameter clear bore, the plant is loaded horizontally inside a cradle positioned at the center of the high homogeneity spherical volume, see Fig. S1A and S1D. The scanner was located in an air-conditioned room $\left(20^{\circ} \mathrm{C}\right)$. WVA106 plants were transferred from the glasshouse to an indoor growth chamber at least one week before MRI measurements in order to adapt to a $12 \mathrm{~h}$-daylight period (PPFD $\sim 300 \mu \mathrm{mol} \mathrm{m}^{-2} \mathrm{~s}^{-1}$ ) and $22^{\circ} \mathrm{C}$ temperature conditions. For MRI experiments, an openable saddle-coil designed for measuring samples up to $14 \mathrm{~mm}$ diameter was installed around the tomato stem (Fig. S1B, C, D). A flip-flop pulse sequence based on slice selectivity and nuclear magnetization relaxation in the context of a multi spin echo sequence (Buy et al., 2018) was applied to measure the xylem fluxes in the active areas of the main plant stem, i.e. areas in which flow was measured. Two pulse sequences measuring inflow and outflow weighted images of the same slice were performed, allowing slow flow sensitive imaging. The mean flux velocity was assessed by fitting the experimental data with the following expression 
$F F_{\text {signal }}=r \cdot M_{0} \cdot \exp \left(-\frac{\mathrm{TR}}{T_{1}}\right)(1)$

Where $\mathrm{M}_{0}$ represents the total magnetization, $\mathrm{T} 1$ the longitudinal relaxation time (s) and $\mathrm{r}$ the inflow factor which depends on sap flow hypothesis (Buy et al., 2018). Using a Poiseuille model, it can be shown that for a laminar flow, $\mathrm{r}$ is proportional to the mean velocity $v_{\text {mean }}$, the repetition time TR ( $\mathrm{s}$ ) and the inverse of the slice thickness. Matlab routines were developed to process MRI measurements at different TR values providing a map of fluxes and mean velocities in the active surfaces of vascular tissues. A particular attention was paid to registered inflow and outflow images before computing the various maps. This method has been previously calibrated on tubes with known diameter and water velocity (Buy et al., 2018) and improved for plants (Simon et al., unpublished).

The MRI measurements were performed on two WVA106 tomato plants of $200 \mathrm{~cm}$ height and bearing 9 trusses, and at three different heights along the main stem: the bottom (as close as possible to the soil), middle (below the fifth truss, bearing expanding fruits) and the top of the stem (between the eight and the ninth flowering truss). For each position and each plant, the stem part that was in the coil was sampled after MRI measurements and processed through the histological pipeline.

During MRI experiments, the leaves emerging outside the bore were illuminated by LED lamps (Alpheus, Montgeron, France) at an intensity of $200 \mu \mathrm{mol} \mathrm{m} \mathrm{m} \mathrm{s}^{-1}$. In order to check how the horizontal position affects the plant, an additional experiment was performed in which the plant was placed outside the magnet, but in similar conditions (horizontal, same light intensity and $\mathrm{T}^{\circ} \mathrm{C}$ ) as in the magnet, and the leaf photosynthetic activity, transpiration and stomatal conductance were measured. The transpiration was low, but stomata were still open (results are described on Fig. S2).

\section{5 - Virtual Fruit model}

The Virtual Fruit was used to assess the potential impact of vascular tissues on fruit growth. This processbased model simulates the water and dry matter accumulation during fruit growth, considering the fruit as one big cell connected to the stem by the pedicel vascular tissues (Fishman and Génard, 1998 ; Liu et al., 2007 ; Hall et al., 2013). Water and sucrose move from the stem to the fruit through the pedicel vasculature, and then within the fruit through composite membranes. Changes in fruit water content over time are calculated as the sum of xylem and phloem net influx (including possible xylem backflow), minus the water outflow due to fruit transpiration.

In the pedicel, the phloem flow depends on the pedicel phloem conductance (Lp1) and the stem-to-fruit gradients of turgor pressure and osmotic potential (Hall et al., 2013). The xylem flow is driven by the hydrostatic pressure difference between the stem xylem and the fruit xylem and depends on the pedicel xylem conductance (Lx1). In previous studies, Lp1 and Lx1 were assumed to remain constant during pedicel and fruit growth. In the present study, they were re-estimated based on the measured phloem and xylem areas.

In the fruit, the xylem and phloem fluxes are calculated from non-equilibrium thermodynamic equations involving the surface of exchange, which is assumed to be proportional to the fruit surface area. It also involves hydraulic conductivity, solute reflection coefficients of the membrane separating the fruit from the vascular tissues of the pedicel and hydrostatic and osmotic pressures, calculated from the molar concentrations of osmotically active compounds (soluble sugars, organic acids, potassium ... ) (Liu et al., 2007).

The model inputs are two measured climatic variables: air temperature and humidity; and two plant variables: stem water potential and phloem sap concentration in sugars, of which values were taken from Constantinescu et al. (2016).

The Virtual Fruit model was calibrated for the genotype WVA106 based on independent data of fruit dry and fresh weights collected on control plants grown in a glasshouse. The nine estimated parameters are involved (1) in the sugars active uptake (nuM, the maximal uptake and tstar, $\tau_{\mathrm{a}}$ giving the pattern of the 
sugar decrease during fruit growth), (2) in the fruit plasticity ( $\Phi_{\max }$, the maximal plasticity and $\mathrm{k}$, giving the plasticity decrease with fruit age) and (3) the xylem and phloem conductance both in the pedicel and in the fruit. The genetic algorithm NSGA-II (Deb et al., 2002) was used to find the combination of parameters, which best predicts the experimental dry and fresh weights. The outputs of this algorithm depend on the random initial values chosen, so the optimization process was repeated 10 times.

\section{6 - Statistical analysis}

Kinetic and histological data were analyzed with the $\mathrm{R}$ software (R Core Team, 2017). Kruskal-Wallis tests were used to compare the relative areas of vascular tissues, the pedicel diameter, the fruit fresh mass and dry matter content, between control and the different WD treatments. Tukey's range tests were used to compare the relative area of the vascular tissues between different plant organs. The flow-MRI data processing and analysis were performed under Matlab programming language (MATLAB, 2016).

\section{3 - RESULTS}

3.1 - Histological quantification of vascular tissues along tomato pedicel

The histological pipeline described above was first applied on pedicels of two genotypes, Heinz wild-type and Heinz Jointless, that differed by the presence (wild-type) or absence (Jointless) of an abscission zone (AZ). The increase in pedicel diameter over fruit development was not significantly different between AZf and AZs (data not shown). Similarly, the final fruit diameter, pedicel diameter and dry matter content (DMC) did not differ significantly between the mutant and the wild-type.

The histological properties along the pedicel (Fig. 1A) were measured at fruit maturity for the 2 genotypes (Fig. 2). At the AZ level, the xylem tissues were clearly non-lignified in the wild-type and the area of each tissue could not be quantified (Fig. 2A). On the contrary in the mutant, a continuous lignification of the secondary xylem was observed at the level of the theoretical AZ (defined by the pedicel curvature) (Fig. 2B). Comparing AZf and AZs, the proportion of secondary xylem to the pedicel section was lower on the fruit side than on the stem side whereas the proportion of cortex was higher. This was observed both in the wild type (Fig. 2C) and the mutant (Fig. 2D), but differences were significant for the wild type only ( $\mathrm{P}=0.013)$. In the mutant, the proportions of tissues in the theoretical AZ and in the AZs were very similar.

In the following, the genotypic variability and environmental impact on tissue proportions were measured on the AZf only for the sake of simplicity.

\section{2 - Assessment of the genotypic variability of pedicel diameter and vascular tissue areas}

The pedicel growth was investigated in relation to fruit expansion on the two Heinz genotypes and on the eight MAGIC TOM parents. Whatever the genotype, the increase in pedicel diameter followed similar trajectories and stopped growing as the fruit was still rapidly expanding (Fig. 3). These observations suggest that the vascular tissue area was set before the occurrence of the maximum fruit growth rate.

A histological analysis of the pedicel was performed on 11 genotypes (the previous 10 genotypes, adding WVA106) at fruit maturity (red ripe stage). Genotypes were grouped in three classes of fresh mass (FM) (Fig. 4A): small fruit $(\mathrm{FM}<15 \mathrm{~g})$, large fruits $(\mathrm{FM}>100 \mathrm{~g})$, and medium fruits in between. The comparison of the three classes outlined significant differences in FM $(\mathrm{P}<0.01)$. The DMC (Fig. 4B) and pedicel diameter (Fig. 4C) were significantly lower in the small fruit class only $(\mathrm{P}<0.01)$.

When expressed in relative proportions of the pedicel section, the cortex was always the most important tissue (more than $30 \%$ of the total area for all genotypes) followed by the secondary xylem or by pith depending on genotype, whereas the external and internal phloem proportions were the smallest (Fig. 4D). Focusing on vascular tissues, the proportion of secondary xylem varied from 15\% (Cervil) to $31 \%$ (Heinz Jointless), the proportion of external phloem varied from $6 \%$ (Cervil) to $11 \%$ (Stupicke) and the proportion of internal phloem varied from $2 \%$ (Cervil) to $7 \%$ (LA0147). In absolute area, the secondary xylem was always the largest and the internal phloem was the smallest (Fig. 4E). The mean absolute area of secondary xylem varied by a factor of 4.3 between WVA106 (lowest value) and Heinz Jointless (highest value), the mean area 
of external phloem varied by a factor of 4.8 between LA1420 and Heinz wild-type and the area of internal phloem varied by a factor of 6.2 between Criollo and Heinz wild-type. To better explore the links among variables, a principal component analysis (PCA) was performed on the proportions of tissues, the pedicel diameter, the fruit FM and DMC (Fig. 5). The first axis (43\% explained variance) separates individuals with high fruit FM, high pith and internal phloem proportions and high pedicel diameter (PCA1>0), from individuals with high proportions of cortex and high DMC $(\mathrm{PCA} 1<0)$. The second axis $(23 \%$ explained variance) mostly represents individuals with high proportions of secondary xylem and external phloem $(\mathrm{PCA} 2<0)$. The projection of the 11 genotypes on the first plane evidenced a first group located at the top right corner and composed of two large fruit genotypes (Levovil and LA0147) and one medium fruit genotype (Heinz wild-type) with relatively low DMC $(<7 \%)$. This group was associated with a low cortex proportion, high pedicel diameter and high pith and internal phloem proportions. On the opposite at the top left corner, Cervil the genotype with the smallest FM and highest DMC was associated with a small pedicel diameter, a high cortex proportion, and low proportions of pith, secondary xylem and internal and external phloems. Two other atypical genotypes were discriminated on the second axis. Ferum, a genotype of the large fruits group with a relatively high DMC was associated with a low proportion of external phloem and secondary xylem, in contrast with LA1420, a genotype with a relatively low DMC compared to its small size $(\mathrm{FM}<25 \mathrm{~g})$. The other small fruit genotypes were poorly represented on this PCA.

3.3 - Water deficit impact on the pedicel diameter and vascular tissues of the tomato pedicel in relation to fruit growth

Three scenarios of WD were applied on genotype WVA106. All of them significantly reduced the fruit FM $(-12.9 \%,-22.6 \%$ and $-26.9 \%$, for the early light, early severe and late severe WD, respectively) and significantly increased the DMC $(+24.3 \%,+24.3 \%$ and $+16.5 \%$, for the early light, early severe and late severe WD, respectively) (Fig. 6A, B). The pedicel diameter was significantly lower in the early severe WD than in the control treatment $(-21.1 \%)$ and was not significantly affected in the two other scenarios (Fig. $6 \mathrm{C})$.

The early severe WD provoked a significant reduction of the absolute areas of secondary xylem $(-54 \%)$, pith (-63\%), external phloem (-55\%) and internal phloem (-51\%). This led to a significant decrease in the proportions of secondary xylem $(-18.6 \%)$ and pith $(-33.3 \%)$, and a significant increase in cortex proportion $(+21.3 \%)$, whereas the phloem tissues were not affected. In this treatment, the strong reduction of the pedicel diameter could explain the significant effect on the absolute areas (Fig. 6C). The early light WD provoked a significant reduction of the absolute areas of pith compared to the control (-53\%). In proportions of the pedicel section, the early light WD significantly increased the proportion of cortex $(+12.8 \%)$ and decreased the proportions of pith (-39.4\%) and internal phloem (-16.6\%). The late WD did not significantly affect the areas of tissues.

3.4 - Model-based analysis of the contribution of vascular tissues to water deficit impact and to genotypic variability on tomato fruit mass

The Virtual fruit model, calibrated for WVA106 in well-watered conditions (see material and methods) was run for a 28-days period of rapid fruit expansion (starting at 10 days after anthesis, i.e. after the cell division period). The final fruit FM and DMC simulated for WVA106 under control conditions were $9.86 \mathrm{~g}$ and $8.3 \%$, respectively, which fits well with the experimental data (Fig. S3). In order to evaluate the contribution of the WD-induced and genotypic variations of xylem and phloem areas on fruit growth, the pedicel conductance were modified in the model according to experimental measurements, all other parameters being unchanged. For that, the phloem and xylem conductances in the pedicel (Lp1 and Lx1) were assumed to be proportional to the xylem and phloem areas measured in this study (Fig. 4D and 6D). Since the model does not distinguish the internal and external phloem, the two measured areas were added.

Therefore, for the early light WD, Lx1 and Lp1 were reduced by $23 \%$ and $36 \%$, respectively according to the reduction of xylem and phloem areas (Fig. 6D). For the early severe WD, both Lx1 and Lp1 were decreased by $54 \%$. For the late severe WD, Lx1 and Lp1 were respectively decreased by $28 \%$ and $27 \%$. The simulated 
FM at maturity was $8.8 \mathrm{~g}$ in the early light WD scenario (experimental data: $8.1+/-0.4 \mathrm{~g}$ ), $7.7 \mathrm{~g}$ in the early severe WD scenario (experimental data: $7.2+/-0.5 \mathrm{~g}$ ) and $8.9 \mathrm{~g}$ in the late severe WD scenario (experimental data: $6.8+/-0.6 \mathrm{~g})$. Thus, in the early light and early severe WD, changes in Lx1 and Lp1 led to a $10 \%$ and $23 \%$ reduction of the simulated fruit FM, respectively, which was similar to the observed reduction $(-13 \%$ and $-23 \%$ respectively). On the opposite, under late severe WD, the reduction of the simulated FM $(-9.6 \%)$ was about three times lower than that measured (-26.8\%). The simulated final DMC was $8.3 \%$ in the control, $8.4 \%$ in the early light WD scenario, $8.9 \%$ in the early severe WD scenario and $8.6 \%$ in the late severe WD scenario. According to the Virtual Fruit model, the reduction of the xylem and phloem conductance at the pedicel level hardly impacted the simulated DMC for the three scenarios whereas an increase of $24 \%$ (early light WD), 23\% (early severe WD) and 16\% (late severe WD) was experimentally measured.

To account for the genotypic variability, we reported on Lx1 and Lp1 the maximum variations of xylem and phloem areas observed between WVA106 and each of the 11 genotypes (Fig. 4D): variations in xylem and phloem areas ranged from 0 to $+332 \%$ and from $-6 \%$ to $+347 \%$, respectively. Therefore, a $10 \%$ decrease and a $350 \%$ increase of Lx1 and Lp1 were applied in the model. The 10\% reduction did not strongly affect the FM and DMC at maturity (9.54 g and 8.4\%, respectively). On the contrary, the $350 \%$ increase of Lx1 and Lp1 increased the simulated FM by $54 \%$ (15.24g compared to $9.86 \mathrm{~g}$ ) and decreased the DMC by $25 \%$ (6.2\% compared to 8.3\%). Thus, according to the Virtual Fruit model, the genotypic variability in Lx1 and Lp1 deduced from the observed variability in vascular tissue areas, contributed very modestly to the actual genotypic variations in fruit FM and DM (Fig. 4D).

\section{5 - Variability of the vascular tissue anatomy within the plant architecture}

The vascular anatomy was investigated in one genotype (WVA106) at different positions within the plant: at three heights along the main stem (bottom, middle and top), at the truss peduncle and at the pedicel AZf levels (Fig. 7).

The proportion of cortex was significantly lower $(\mathrm{P}<0.01)$ in the stem than in the peduncle and pedicel, and the proportion of pith was significantly higher in the stem than in the pedicel $(\mathrm{P}<0.01)$. At the main stem level, the proportion of secondary xylem decreased significantly $(\mathrm{P}<0.05)$ from the bottom to the top of the plant (Fig. 7A). At the bottom of the stem, the internal phloem proportion was significantly lower $(\mathrm{P}<0.01)$ and the external phloem was significantly higher $(\mathrm{P}<0.01)$ than at the middle and the top where the external and internal phloem proportion were similar. The absolute areas of all tissues, except the internal phloem, decreased from the bottom to the top of the plant (Fig. 7B), in relation with a decrease in stem diameter.

An image analysis of the histological slices allowed to quantify the number and dimensions of xylem vessels in the peduncle and main stem (Table 1) and to estimate a theoretical xylem conductance based on the vessel diameter distributions and the Hagen-Poiseuille relation (Calkin et al., 1985):

$k_{h}=\left(\frac{\pi}{128 \eta}\right) \sum_{i-1}^{n}\left(d_{i}^{4}\right)(2)$

where $\eta$ is the dynamic viscosity of the fluid (MPa s), $d$ the diameter $(\mathrm{m})$ of the $i$ th vessel and $n$ the number of vessels. We considered here that the viscosity of xylem sap was equal to that of water $\left(\eta=110^{-9} \mathrm{MPa} s\right.$ at $20^{\circ} \mathrm{C}$ ). The theoretical conductance of the xylem vessels of the peduncle was around $9.410^{-11} \mathrm{~m}^{4} \mathrm{MPa}^{-1} \mathrm{~s}^{-1}$ (Table 1). In the main stem, the theoretical conductance decreased by one to two orders of magnitude from the bottom to the top of the plant, especially in the upper part due to the low number of vessels (Table 1).

3.6 - MRI investigations of xylem flow along the main stem

The variability of xylem tissues along the stem was further investigated by MRI. Six MRI experiments were conducted on two plants, at three heights of the main stem. The results are reported for plant 1 and 2 in Figure 8 and S4, respectively. From flow-MRI experiments, a significant FF-signal with respect to the signal to noise ratio in the image permits to discriminate between active and passive vessels and the direction of the water transport to the top of the plant. Fits using Eq. 1 of the experimental data obtained from the integrated FF signals over the active vessels (colored in yellow in Fig. 8A and C) at various repetition time 
(TR) allows us to estimate the mean velocities of the flux and the mean flow (see Table 1). For example, the mean flux velocity measured at the bottom of the first plant was $0.259 \mathrm{~mm} \mathrm{~s}^{-1}$ and the area of conducting tissues was $1.6 \mathrm{~mm}^{2}$, giving a water flow of $0.4 \mathrm{~mm}^{3} \mathrm{~s}^{-1}$, which approximatively corresponds to $1.44 \mathrm{~g} \mathrm{~h}^{-1}$.

From a detailed analysis of the inflow MRI images, it was also possible for the first time to identify the active vessels, to measure their dimensions at each stem height and to estimate their theoretical conductances (Table 1). All active vessels were of xylem type. As illustrated with experiments performed at the bottom of the first plant (Fig. 8F), the comparison of the distributions of the diameters measured on the histological slice on all the xylem vessels (green color) with that observed on the inflow MRI image corresponding to the active vessels (red color), indicated that less than half of the xylem vessels were active under the MRI conditions and that these vessels were mainly the largest ones (see Fig. S4B for the middle and top of the stem and for the second plant).

Interestingly, the number and proportion of active vessels decreased from the bottom to the top of the plant, leading to a large decrease of the active theoretical conductance, especially at the top of the stem. The ratio of the number of active vessels to the total number of vessels also decreased from the bottom to the top of the plant. The ratio of the active theoretical conductance to the total theoretical conductance was the lowest at the top of the plant and quite similar at the middle and bottom of the stem. This ratio was way larger than the ratio of the number of active xylem vessels to the total number of xylem vessels. Indeed, the inactive xylem vessels were mainly the smallest ones, and their contribution to the total conductance was limited. These results highlighted that, despite stable flux velocities at different heights of the plant, the xylem flow strongly varied along the stem and between individual plants grown under similar conditions, due to variations in the number and size of the xylem vessels that were mobilized.

\section{4 - DISCUSSION}

Xylem and phloem flows are regulated by pressure gradients in the whole plant system and depend on the anatomical and hydraulic properties of the conducts (Johnson et al., 1992). Although the vasculature area is not considered as the driver of sink growth, it has been shown to have both deterministic and plastic properties and it could be a primary driving force for plant diversification and adaptation to particular growth conditions (Lucas et al., 2013). The genetic and environmental variabilities in vasculature have been rarely described in tomato and more generally in herbaceous plants. In the present study, the first objective was to focus on the genetic and WD-induced variability of fruit pedicel anatomy and assess its contribution to fruit mass variability by coupling histological and modeling approaches. A second objective was to study the links between structural variability and functional properties within the architecture of reproductive plants by combining histological and MRI methods at the stem level.

\section{1 - The decrease in vascular tissue areas along the pedicel is independent of the presence of} an abscission zone

Many studies focused on the hydraulic resistance of the tomato pedicel and its impact on the supply of water and minerals, calcium in particular, to the fruit (Belda and Ho, 1993; Malone and Andrews, 2001; van Ieperen et al., 2003; Rancic et al., 2010). In the present study, a clear discontinuity of the xylem lignification was observed at the abscission point, in agreement with a previous study on this genotype (Szymkowiak and Irish, 1999), together with a decrease of the xylem proportion along the pedicel which was independent of the presence or absence of AZ. Despite the absence of AZ, the Jointless mutant had smaller fruit FM and a higher DMC, which is counter-intuitive since the hydraulic resistance along the pedicel should be lower for the mutant than for the wild-type (van Ieperen el al., 2003). However, the mutant had a smaller pedicel diameter compared to the wild-type with similar proportion of tissues. So the lower areas of xylem and phloem tissues may contribute to the lower fruit FM of the mutant, although other factors may be involved as well. Altogether, these results support the hypothesis that the non-lignification of tissues in the AZ does not necessarily reduce the hydraulic conductivity and that only the mechanical components of the xylem (fibres and xylem parenchyma) may be interrupted (Malone and Andrews, 2001). They also suggest that the increasing hydraulic resistance along the pedicel mainly results from the decreasing area of conductive 
tissues in agreement with previous studies (Lee, 1989; André et al., 1999; Rancic et al., 2010).

\section{2 - Pedicel anatomical traits are worth being phenotyped for screening genetic resources}

Constantinescu et al. (2016) suggested that pedicel conductivity and active sugar uptake could be interesting traits to maintain FM of large-fruit genotypes under WD and increase their DMC under well-irrigated conditions. In the present study, a large genetic variability in the absolute surface of xylem and phloem was observed, which has been rarely reported before. Apart from Heinz genotypes, the larger the pedicel diameter and tissue areas, the larger the fruit mass. According to the Virtual Fruit Model, the variations in xylem and phloem areas alone could not account for the 100-fold difference in FM between cherry tomatoes and large fruits, but it could partly contribute. In proportion, the relative areas of tissues varied by a factor 2 to 3 among genotypes, the internal phloem being the most variable tissue. In tomato, fruit trusses are mainly supplied by the two leaves below the truss through upward movement of assimilates in the internal phloem (Bonnemain, 1968), which is in line with the positive correlation observed between internal phloem proportion and FM (Fig. 5). They are also consistent with the assumption that most water enters the fruit through the phloem tissues (Ho et al., 1987; Guichard et al., 2005). On the contrary no clear relation was observed between the proportion of secondary xylem and the fruit FM or DMC. This seems to be in contradiction with the reduction of xylem production and lignification reported in the AZs of pedicels from mature cherry like tomatoes compared to large-fruit cultivars (André et al., 1999).

\section{3 - The effect of water deficit on the pedicel anatomy and areas of vascular tissues partially explains its effects on fruit mass and dry matter content}

Although many factors, such as stem water potential, sap sugar concentration and viscosity, osmoticum accumulation, etc., are involved in fruit water balance under WD (Ho et al., 1987; Bussières, 2002 ; Ripoll et al., 2014; 2016), the present study was designed to assess the contribution of the pedicel anatomy. The early WD treatments affected the whole period of fruit development including cell division, while the late WD treatment impacted mostly the cell expansion period. Indeed in WVA106, cell division ends about 10 daa, which corresponds to the first third of the development period (Renaudin et al., 2017). Assuming that the increase in pedicel diameter reflects the production of new vessels during secondary growth (van Ieperen et al., 2003), then according to Fig. 3, the number of vessels was fixed before fruit cell division ceased, and much before the maximum fruit growth rate. This is confirmed by the reduction of both pedicel diameter and vascular tissues areas under the early severe WD, but not under the late severe WD. So under early severe $\mathrm{WD}$, the reduction in xylem and phloem areas would have contribute significantly to the decrease in fruit FM, which was related to the intensity of stress rather than to the period of application. The model-based analysis confirmed that the observed reduction in xylem and phloem areas alone could account for most of the reduction of fruit FM observed under early WD. On the contrary, the increase in DMC was more sensitive to the period of WD and could result from changes in tissue proportions rather than in absolute areas. Indeed in case of early WD the proportions of tissues were affected at both intensities with more cortex and less pith, but also less internal phloem and secondary xylem for the light and severe water deficits, respectively. So the model should also take into account the effect of WD on the proportions of xylem and phloem. In agreement, Hanssens et al. (2015) showed that the relative xylem contribution to tomato fruit growth is strongly affected by environmental conditions. Moreover the xylem conductance depends on the diameter of individual vessels rather than on the whole surface of xylem tissue as assumed in the modeling approach. Several studies showed that WD decreased the diameter of xylem vessels, in tomato pedicel (Rancic et al., 2008), in grape (Lovisolo and Schubert, 1998) and in maize (Abdel-Rahim et al., 1998; Liu et al., 2019) or in poplar tress (Arend and Fromm, 2007). In tomato plants grown under WD, the conductance decreases in the pedicel AZ, but is increased on both sides due to new vessel formation (van Ieperen et al., 2003). Such variations may greatly affect the tissue conductance, and outlined the need to investigate and model the number of conducting elements and their diameter distribution, rather than the total conductive area.

4.4 - Histological and MRI investigations of xylem anatomical and hydraulic properties along the stem 
In the main stem, xylem provides mechanical support and transports water, mineral and hormones from the root to the different organs. Predictive models usually consider homogeneous anatomical and hydraulic properties within the plant architecture. However, height- and position-related variations in the number and size of xylem vessels have been linked to variations in segment-specific resistance in tree architecture (Soriano et al., 2020). In tomato, Qi et al. (2020) reported a decrease in xylem proportion from the bottom to the top of the stem of 6 weeks tomato plants and attributed yield differences between Dutch and Japanese cultivars to the cytokine-driven development of secondary xylem in the stem of young vegetative plants. In the present study, MRI and histological measurement performed on reproductive plants, confirmed the sharp decrease in xylem area from the bottom to the top of the stem (Fig. 7) both in absolute and relative values, whereas the proportion of internal phloem increased to the top of the plant where it was similar to that observed in peduncle and pedicel. The low proportion of internal phloem tissues at the bottom of the stem may be related to the small flow of photosynthates moving towards the roots at this stage. MRI measurements were performed on a sufficiently high and homogeneous magnetic field of $9.4 \mathrm{~T}$ with a resolution of $20 \mu \mathrm{m}$ x $20 \mu \mathrm{m}$ in plane, allowing to observe in details xylem vessels. Our results indicated that only part of the xylem vessels were active i.e contributed to the measured flow, and the ratio of active vessels decreased 3 -fold from the bottom to the top of the stem (Table 1). This result is consistent with MRI images of flow conducting tissues in tomato peduncle (Windt et al., 2009). As shown on Fig. 8, the active vessels were the largest ones even though a large number of tiny vessels were visible over the whole stem section. The low ratio of active xylem vessels likely indicated that the low evaporative demand under the MRI experimental conditions (Fig. S2) was easily supplied by a few large vessels. In the future, it will be interesting to look for an adaptation of the number of active vessels at constant water flow velocity to the environmental conditions during MRI experiment. For instance, the low light intensity may have decrease the total sap flow and increase the relative contribution of xylem to fruit water import as evidenced by Hanssens et al. (2015). Xylem cavitation leading to the presence of air in the vessels could be another artefact, but it would have been easily identified by MRI. Other xylem blockages could occur as observed in grape stem and berry pedicel, but this does not preclude xylem functionality due to interconnections (Knipfer et al., 2015).

In the MRI conditions experimented by the plant, the theoretical xylem conductance varied by one to two order of magnitudes along the stem and these variations resulted mainly from the number of vessels. To our knowledge, no value of conductance has been reported at the stem level of tomato plant, but the values estimated in Table 1 are of the same order of magnitude than conductance values measured in maize roots (Doussan et al., 1998) and in trees (Cruiziat et al., 2002). Our values measured in tomato stem are higher than the conductance estimated at the pedicel level by Rancic et al. (2010), which makes sense since the xylem area was higher in the stem than in the pedicel (Fig. 7B). The large active vessels contributed for most of the theoretical conductance (Table 1), which suggests that investigating only the largest vessels may be sufficient to estimate the tissue conductance.

\section{5 - Concluding remarks and perspectives}

The present study highlights the interest to combine flow-MRI with histological and modeling approaches for a better understanding of hydraulic regulations in the architecture of intact plants and in response to genotypic and environmental variations. The proportion of active vessels and the distributions of vessel diameter throughout the plant architecture appeared as major factors to improve the predictive capacity of plant models.

\section{AUTHOR CONTRIBUTION}

M. C., B. B., E. A., C. B. and J. S. carried out experiments. C. G.-B., N. B. and J. S. designed the project. M. C., M. L., G. V., J.-L. V. and J. S. contributed to data analysis. N. B., C. G.-B. and J. S. wrote the manuscript. M. C., G. V. and J.-L. V. proofread the manuscript.

\section{ACKNOWLEDGEMENTS}

We thank Guillaume Garcia, Thibault Crouzet and Pierre Valsesia for their valuable contributions to this work. 


\section{CONFLICT OF INTEREST}

The authors declare no conflict of interest.

\section{References}

Abd El-Rahim M. F., Fahmy G. M., \& Fahmy Z. M. (1998). Alterations in transpiration and stem vascular tissues of two maize cultivars under conditions of water stress and late wilt disease. Plant pathology, 47(2), 216-223.

André J. P., Catesson A. M., \& Liberman M. (1999). Characters and origin of vessels with heterogenous structure in leaf and flower abscission zones. Canadian Journal of Botany , 77(2), 253-261.

Arend M. \& Fromm J. (2007). Seasonal change in the drought response of wood cell development in poplar. Tree physiology, 27(7), 985-992.

Belda R. M. \& Ho L. C. (1993). Salinity effects on the network of vascular bundles during tomato fruit development. Journal of horticultural science, 68(4), 557-564.

Bonnemain J. L. (1968). Anatomie et physiologie de l'appareil conducteur des Solanacées (Doctoral dissertation).

Bussières P. (2002). Water import in the young tomato fruit limited by pedicel resistance and calyx transpiration. Functional Plant Biology, 29(5), 631-641.

Bussières P., Bertin N., Morris C. E., Vigne C., Orlando P., Glaux C., Sévenier V., Floret H., Bernadac J. \& Korownikoff S. (2011). High external sucrose concentration inhibits the expansion of detached tomato fruits grown in a novel semi-open device. In Vitro Cellular 83 Developmental Biology-Plant, 47(6), 743-751.

Buy S., Le Floch S., Tang N., Sidiboulenouar R., Zanca M., Canadas P., Nativel E., Cardoso M., Alibert E., Dupont G., Ambard D., Maurel C., Verdeil J.-L., Bertin N., Goze-Bac C. \& Coillot C. (2018). Flip-flop method: A new T1-weighted flow-MRI for plants studies. PLoS One,13(3).

Calkin H. W., Gibson A. C., \& Nobel P. S. (1985). Xylem water potentials and hydraulic conductances in eight species of ferns. Canadian Journal of Botany, 63(3), 632-637.

Constantinescu D., Memmah M. M., Vercambre G., Génard M., Baldazzi V., Causse M., Albert E., Brunel B., Valsesia P. \& Bertin N. (2016). Model-assisted estimation of the genetic variability in physiological parameters related to tomato fruit growth under contrasted water conditions. Frontiers in plant science, 7 , 1841.

Constantinescu D., Vercambre G., \& Génard M. (2020). Model-assisted analysis of the peach pedicel-fruit system suggests regulation of sugar uptake and a water-saving strategy. Journal of Experimental Botany, 71(12), 3463-3474.

Cruiziat P., Cochard H., \& Améglio T. (2002). Hydraulic architecture of trees: main concepts and results. Annals of forest science, 59(7), 723-752.

Deb K., Pratap A., Agarwal S. \& Meyarivan T. A. M. T. (2002). A fast and elitist multiobjective genetic algorithm: NSGA-II. EEE transactions on evolutionary computation, 6(2), 182-197.

Doussan C., Vercambre G., \& Pagès L. (1998). Modelling of the hydraulic architecture of root systems: An integrated approach to water absorption-Distribution of axial and radial conductances in maize.Annals of Botany , 81(2), 225-232.

Fishman S. \& Génard M. (1998) A biophysical model of fruit growth: simulation of seasonal and diurnal dynamics of mass. Plant, Cell and Environment, 21(8), 739-752.

Guichard S., Bertin N., Leonardi C. \& Gary C. (2001). Tomato fruit quality in relation to water and carbon fluxes. 
Guichard S., Gary C., Leonardi C. \& Bertin N. (2005). Analysis of growth and water relations of tomato fruits in relation to air vapor pressure deficit and plant fruit load. Journal of Plant Growth Regulation, 24(3), 201.

Hall A. J., Minchin P. E. H., Clearwater M. J. \& Génard M. (2013). A biophysical model of kiwifruit (Actinidia deliciosa) berry development.Journal of Experimental Botany, 64(18), 5473-5483.

Hanssens J., De Swaef T. \& Steppe K. (2015). High light decreases xylem contribution to fruit growth in tomato. Plant, cell \& environment, 38(3), 487-498.

Heuvelink E. (1995). Dry matter partitioning in a tomato plant: one common assimilate pool? Journal of Experimental Botany , 46(8), 1025-1033.

Ho L. C., Grange R. I. \& Picken A. J. (1987). An analysis of the accumulation of water and dry matter in tomato fruit. Plant, Cell \& Environment, 10(2), 157-162.

Ho L. C. (1996). The mechanism of assimilate partitioning and carbohydrate compartmentation in fruit in relation to the quality and yield of tomato. Journal of Experimental Botany, 1239-1243.

Johnson R. W., Dixon M. A., \& Lee D. R. (1992). Water relations of the tomato during fruit growth. Plant, Cell \& Environment, 15(8), 947-953.

Knipfer T., Eustis A., Brodersen C., Walker A. M., \& McElrone A. J. (2015). Grapevine species from varied native habitats exhibit differences in embolism formation/repair associated with leaf gas exchange and root pressure. Plant, Cell \& Environment, 38(8), 1503-1513.

Koch G., Rolland G., Dauzat M., Bédiée A., Baldazzi V., Bertin N., Guédon Y. \& Granier C. (2019). Leaf Production and Expansion: A Generalized Response to Drought Stresses from Cells to Whole Leaf Biomass-A Case Study in the Tomato Compound Leaf. Plants,8(10), 409.

Lee D. R. (1989). Vasculature of the abscission zone of tomato fruit: implications for transport. Canadian Journal of Botany, 67(6), 1898-1902.

Liu H. F., Génard M., Guichard S. \& Bertin N. (2007). Model-assisted analysis of tomato fruit growth in relation to carbon and water fluxes. Journal of Experimental Botany, 58(13), 3567-3580.

Liu J., Kang S., Davies W. J. \& Ding R. (2019). Elevated [CO2] alleviates the impacts of water deficit on xylem anatomy and hydraulic properties of maize stems. Plant, cell $\& 3$ environment, 43.

Lough T. J. \& Lucas W. J. (2006). Integrative plant biology: role of phloem long-distance macromolecular trafficking. Annual Reviews of Plant Biology, 57, 203-232.

Lovisolo C. \& Schubert A. (1998). Effects of water stress on vessel size and xylem hydraulic conductivity in Vitis vinifera L. Journal of Experimental Botany, 49(321), 693-70 0.

Lucas W. J., Groover A., Lichtenberger R., Furuta K., Yadav S. R., Helariutta Y., He X.-Q., Fukuda H., Kang J., Brady S. M., Patrick J. W., Sperry J., Yoshida A., Lopez-Millan A.-F., Grusak M. A. \& Kachroo P. (2013). The plant vascular system: evolution, development and functions f. Journal of integrative plant biology , 55 (4), 294-388.

Malone M. \& Andrews J. (2001). The distribution of xylem hydraulic resistance in the fruiting truss of tomato. Plant, Cell \& Environment, 24(5), 565-570.

Mao L., Begum D., Chuang H. W., Budiman M. A., Szymkowiak E. J., Irish E. E. \& Wing R. A. (2000). JOINTLESS is a MADS-box gene controlling tomato flower abscission zone development. Nature, 406(6798), 910-913.

MATLAB (2016). Version 9.0.0 (R2016a) . Natick, Massachussetts: The MathWorks Inc. 
Pascual L., Desplat N., Huang B. E., Desgroux A., Bruguier L., Bouchet J. P., Le G. H., Chauchard B., Verschave P. \& Causse M. (2015). Potential of a tomato MAGIC population to decipher the genetic control of quantitative traits and detect causal variants in the resequencing era. Plant biotechnology journal, 13(4), 565-577.

Qi X., Takahashi H., Kawasaki Y., Ohta Y., Isozaki M., Kojima M., Takebayashi Y., Sakakibara H., Imanishi S., Chen X. \& Nakazono M. (2020). Differences in xylem development between Dutch and Japanese tomato (Solanum lycopersicum) correlate with cytokinin levels in hypocotyls. Annals of Botany .

Rančić D., Quarrie S. P., Terzić M., Savić S. \& Stikić R. (2008). Comparison of light and fluorescence microscopy for xylem analysis in tomato pedicels during fruit development. Journal of microscopy,232(3), 618-622.

Rančić D., Quarrie S. P. \& Pećinar I. (2010). Anatomy of tomato fruit and fruit pedicel during fruit development. Microscopy: Science, Technology, Applications and Education, 2, 851-861.

Renaudin J. P., Deluche C., Cheniclet C., Chevalier C., \& Frangne N. (2017). Cell layer-specific patterns of cell division and cell expansion during fruit set and fruit growth in tomato pericarp. Journal of experimental botany , 68(7), 1613-1623.

R Core Team (2017). R: A language and environment for statistical computing. R Foundation for Statistical Computing, Vienna, Austria.

Ripoll J., Urban L., Staudt M., Lopez-Lauri F., Bidel L. P., \& Bertin N. (2014). Water shortage and quality of fleshy fruits - making the most of the unavoidable. Journal of Experimental Botany ,65(15), 4097-4117.

Ripoll J., Urban L., \& Bertin N. (2016). The potential of the MAGIC TOM parental accessions to explore the genetic variability in tomato acclimation to repeated cycles of water deficit and recovery. Frontiers in plant science, 6, 1172.

Schindelin J., Arganda-Carreras I., Frise E., Kaynig V., Longair M., Pietzsch T., Preibisch S., Rueden C., Saalfeld S., Schmid B., Tinevez J.-Y., White D. J., Hartenstein V., Eliceiri K., Tomancak P. \& Cardona A. (2012). Fiji: an open-source platform for biological-image analysis. Nature methods, 9(7), 676-682

Sharma A. K. (1956). Fixation of plant chromosomes. The Botanical Review, 22(10), 665-695.

Slack G., \& Calvert A. (1977). The effect of truss removal on the yield of early sown tomatoes. Journal of Horticultural Science, 52(2), 309-315.

Soriano D., Echeverría A., Anfodillo T., Rosell J. A., \& Olson M. E. (2020). Hydraulic traits vary as the result of tip-to-base conduit widening in vascular plants. Journal of Experimental Botany,71(14), 4232-4242. Spiegelman Z., Ham B. K., Zhang Z., Toal T. W., Brady S. M., Zheng Y., Fei Z., Lucas W. J. \& Wolf S. (2015). A tomato phloem-mobile protein regulates the shoot-to-root ratio by mediating the auxin response in distant organs. The Plant Journal, 83(5), 853-863.

Szymkowiak E. J. \& Irish E. E. (1999). Interactions between jointless and wild-type tomato tissues during development of the pedicel abscission zone and the inflorescence meristem. The Plant Cell,11(2), 159-175.

Tolivia D. \& Tolivia J. (1987). Fasga: a new polychromatic method for simultaneous and differential staining of plant tissues. Journal of Microscopy, 148(1), 113-117.

Van As H. (2007). Intact plant MRI for the study of cell water relations, membrane permeability, cell-to-cell and long distance water transport. Journal of Experimental Botany, 58(4), 743-756.

Van Bel A. J. E. (1990). Xylem-phloem exchange via the rays: the undervalued route of transport. Journal of Experimental Botany,41(6), 631-644.

Van Ieperen W., Volkov V. S. \& Van Meeteren U. (2003). Distribution of xylem hydraulic resistance in fruiting truss of tomato influenced by water stress. Journal of Experimental Botany, 54(381), 317-324. 
Windt C. W., Gerkema E. \& Van As H. (2009). Most water in the tomato truss is imported through the xylem, not the phloem: a nuclear magnetic resonance flow imaging study. Plant Physiology, 151(2), 830-842.

Figure 1: (A) Flower and pedicel of a WVA106 tomato plant. (B) Schematic representation of a tomato plant with red ripe and growing fruits on several trusses along the stem. Transversal histological sections of a WVA106 tomato (C) fruit pedicel (fruit side or AZf), (D) truss peduncle and (E) main stem. FASGA stained : the red/pink zones indicate the cells with lignified walls (secondary xylem and support fibers) and the blue zones indicate the cells with non lignified cellulosic walls (phloem and parenchyma). The yellow lines correspond to the segmentation of the different tissue types. Segmentation was done using a plugin (PHIV_Tomate_toolset) developed on Fiji allowing to measure the area of each segmented histological zone. For $(\mathrm{C})$ and (E), section thickness: $60 \mu \mathrm{m}$. For (D), section thickness: $80 \mu \mathrm{m}$. For (C) and (D), scale bars: $1 \mathrm{~cm}$. For (E), scale bar: $2 \mathrm{~cm}$.

Figure 2: Transversal histological sections (A and $\mathrm{B})$ and relative proportions of tissues $(\mathrm{C}$ and $\mathrm{D})$ along the tomato pedicel of Heinz wild-type (A and C) and Heinz Jointless (B and D) genotype. (A, B) Left: fruit side of the pedicel (AZf); middle: actual (wild type) or theoretical (jointless: at the level of the pedicel curvature) abscission zone (AZ); right: stem side of the pedicel (AZs). (C, D) The different shades represent the different tissue types (see label box). Each point (in transparency) is an experimental measurement. Error bars represent the standard deviation among fruits. Stars indicate significance differences between the proportions of tissue in the AZs and the AZf: ${ }^{*} \mathrm{p}<0.05,{ }^{* *} \mathrm{p}<0.01$. Transverse sections (thickness: $60 \mu \mathrm{m}$ ) were stained by FASGA. Scale bars: $1 \mathrm{~mm}$.

Figure 3: Pedicel and fruit growth kinetics. (A) Pedicel diameter normalized plant by plant by the mean value of diameter at the plateau as a function of the fruit age normalized by the age at maturity. (B) Fruit diameter normalized plant by plant by the final diameter as a function of the normalized fruit age. For (A) and (B) each point represents one plant at a given time, the different colors represent the different genotypes and the black dashed line indicates the time when pedicels growth reached a plateau. Measurements were carried out on reproductive plants bearing 7 to 10 fruiting trusses.

Figure 4: Fruit and pedicel traits depending on genotypes: (A) Final fruit fresh mass in g. (B) Final fruit dry matter content in $\mathrm{g} 100 \mathrm{~g}^{-1}$. (C) Final pedicel diameter in $\mathrm{mm}$. (D) Mean area proportion of the tissues in the pedicel section. (E) Area of the vascular tissues in the pedicel section in $\mu \mathrm{m}^{2}$. The different shades represent the different tissues (see label box). For (A), (B), (C), (D) and (E), genotypes are ranked from the smaller to the larger genotype in terms of fresh mass, from left to right. Three categories of fruit size are indicated: small fruits $(\mathrm{FM}<15 \mathrm{~g})$, large fruits $(\mathrm{FM}>100 \mathrm{~g})$ and medium fruits between. Each point (in transparency) represents a fruit. Measurements were carried out on reproductive plants bearing 7 to 10 fruiting trusses.

Figure 5: Principal Component Analysis (PCA) of the genetic variations in fruit and pedicel traits. Each point represents the barycenter of the genotype, with error bar representing the standard deviation, and each color represents a genotype. Variables are scaled to unit variance and highly correlated variables have been removed $(>0.85)$. Small fruit: $F M<15 \mathrm{~g}$, large fruits $\mathrm{FM}>100 \mathrm{~g}$ and medium fruits in between. The third axes and the fourth explained $10 \%$ of the variance each, but did not discriminate well the genotypes.

Figure 6: Influence of water deficit (WD) treatments on fruit and pedicel traits: (A) Final fruit fresh mass in g. (B) Final dry matter content in the fruit in g $100 \mathrm{~g}^{-1}$. (C) Final pedicel diameter in mm. (D) Area of tissues in the pedicel section in $\mu^{2}$. (E) Mean area proportions of tissues in the pedicel section. The different shades represent the different tissues (see legend). Early WD occurred 15 days before truss anthesis, and late WD occurred 4 days after the truss anthesis. Light WD means a reduction of $50 \%$ of the well-watered control and severe WD means a reduction of $65 \%$ of the control. Each point (in transparency) represents a fruit. Stars indicate significance differences between the proportion of the tissue in the corresponding treatment and the control treatment: ${ }^{*} \mathrm{p}<0.05,{ }^{*} \mathrm{p}<0.01$. Measurements were carried out on reproductive plants bearing 7 to 10 fruiting trusses.

Figure 7: (A) Mean area proportions of tissues and (B) area of tissues $\left(\mu \mathrm{m}^{2}\right)$ in the main stem (three 
different heights: bottom, middle, top), in the truss peduncle and in the fruit pedicel sections of a WVA106 tomato plant. The different shades represent the different tissues (see legend). Each point (in transparency) represents an experimental measurement.

Table 1: Estimation of the theoretical conductance of all xylem vessels (histological slice) and of active xylem vessels (evidenced on the MRI image) and their associated parameters, for the truss peduncle (mean of 4 plants) and for three heights of the main stem on two plants (WVA106 genotype).

Figure 8: MRI quantification of water flow in the main stem of a tomato plant. (A) and (C) Inflow MRI images of transversal sections at three heights of the main stem of two plants: bottom (as close as possible to the soil), middle (below the fifth truss, bearing expanding fruits) and top (between the eight and the ninth flowering truss) in full-watered conditions. Repetition time: $1200 \mathrm{~ms}$, slice thickness: $0.5 \mathrm{~mm}$ and echo time: $12 \mathrm{~ms}$. The white area represents a high flip-flop signal, corresponding to the active vessels evidenced by flow-MRI measurements. Systematic FF experiments were performed at different repetition times in order to determine the mean flux velocity. The time duration of the flow-MRI experiments depended on the repetition time and lasted from 4 min to 40 minutes, giving a total FF experiments time of 3 hours. Scale bars: $4 \mathrm{~mm}$. (B) and (D) Histological FASGA stained slices of the same parts of both plants. Thickness: $100 \mu \mathrm{m}$. Scale bars: $4 \mathrm{~mm}$. (E) Flip-flop signal as a function of the repetition time in second for the three heights of plant 1 . The symbols represent the measured FF signal at different repetition times and the curve its best fit. (F) Distribution of vessel diameters in $\mu \mathrm{m}$ at the bottom of the first plant. In green: xylem vessels measured on the histological slice. In red: active xylem vessels estimated on the MRI image. Due to the lower resolution of MRI, the size of the largest vessels was slightly overestimated compared to the histological measurement.

Supplementary :

Figure S1:

(A) Agilent MRI scanner working at $9.4 \mathrm{~T}$ with a $10 \mathrm{~cm}$ diameter clear bore. (B) Openable saddle-coil designed for measuring samples up to $14 \mathrm{~mm}$ diameter. (C) The openable saddle coil installed around the main stem of a WVA106 tomato before an MRI experiment. (D) Schematic representation of the MRI experimental set-up with the plant loaded inside a horizontal high superconducting magnetic field. The acquisition is performed at the middle of the main stem. Leaves inside the bore are not represented on the scheme because they are wrapped in a plastic film. Leaves emerging outside the bore are illuminated by

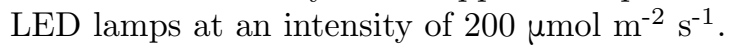

Figure S2:

(A) Leaf photosynthetical activity, (B) transpiration and (C) stomatal conductance depending on leaf rank, in the MRI environmental conditions. Light intensity was set at 100 (red), 180 (green) and 260 (blue) $\mu$ mol $\mathrm{m}^{-2} \mathrm{~s}^{-1}$ which corresponded to the light intensity provided to the leaves outside the magnet during MRI experiments.

Results showed that, at the stage of measurements, the upper leaves were more active than the lower leaves. Compared to greenhouse measurements (not shown), the photosynthetic activity was slightly lower under the MRI conditions, but it was not affected by the horizontal position. The transpiration was low under MRI conditions (between 0.5 and $1.5 \mathrm{mmol} \mathrm{H}_{2} \mathrm{O} \mathrm{m}^{-2} \mathrm{~s}^{-1}$ depending on the leaf rank under MRI conditions compared to about $2.5 \mathrm{mmol} \mathrm{H} \mathrm{O} \mathrm{m}^{-2} \mathrm{~s}^{-1}$ in the greenhouse conditions), but fruits were still growing $(0.06 \mathrm{~g}$ day $^{-1}$ on average over 7 days under MRI conditions compared to $0.19 \mathrm{~g}$ day $^{-1}$ in the greenhouse conditions).

Figure S3: Measured and simulated evolution of fruit dry mass (red), fresh mass (black) and dry matter content (green) of WVA106. Points are experimental measurements and solid lines represent the simulations under control condition. (B) Simulation of three water deficit scenarios with the Virtual fruit model. The dotted lines represent the simulations for the early light WD, with a $23 \%$ decrease of Lx1 and a $36 \%$ decrease of Lp1. The dashed lines represent the simulations for the early severe WD, with a $54 \%$ decrease of Lx1 and Lp1. The dashed and dotted lines represent the simulations for the late severe WD, with a $28 \%$ decrease 
of Lx1 and a $27 \%$ decrease of Lp1. (A) Simulation of the genotypic variability. The dotted lines represent the simulations with a $10 \%$ reduction of Lx1 and Lp1, two model parameters that represent respectively the xylem and phloem conductance in the pedicel. The dashed lines represent the simulations with a $350 \%$ increase of Lx1 and Lp1. For (A) and (B), all the other parameters remain unchanged. The pedicel boundary conditions in the model, i.e. the stem water potential and the sugar concentration in the phloem, varied respectively, from -2 bar at night to -4.6 bar at midday and 0.5 moles of sugar $\mathrm{dm}^{-3}$ sap.

Figure S4:

(A) Flip-flop signal as a function of the repetition time in second for the three heights of the second plant. The symbols represent the measured FF signal at different repetition times and the curve its best fit. (B) Distribution of vessel diameters in $\mu \mathrm{m}$ for the middle and the bottom of the first plant and for the three heights of the second plant. In green: xylem vessels measured on the histological slice. In red: active xylem vessels estimated on the MRI image. Due to the lower resolution of MRI, the size of the largest vessels was slightly overestimated compared to the histological measurement.

\section{Hosted file}

Figures.pdf available at https://authorea.com/users/319610/articles/478111-variability-ofstructural-and-functional-traits-of-vascular-tissues-in-tomato-modeling-histology-andflow-mri-investigations 